\title{
A UNIQUE CASE OF BILATERAL VOCAL FOLD PARALYSIS FOLLOWING SPINAL ANAESTHESIA
}

\author{
Manasij Mitra ${ }^{1}$ \\ drmanasijmitra@gmail.com \\ Nupur Biswas ${ }^{1}$ \\ biswasnupur78@gmail.com \\ Kumar Shailendra \\ kmrshailendra382@gmail.com \\ Anil Chandra Jain \\ Jainanil16@yahoo.com \\ Maitraye Basu \\ Department of Biochemistry ${ }^{2}$ \\ maitraye.basu@gmail.com \\ ${ }^{1}$ Department of Anaesthesiology \\ ${ }^{2}$ MGM Medical College and LSK Hospital, Kishanganj \\ Purab Palli Road, Kishanganj, Bihar, India, 855107
}

\begin{abstract}
Cranial nerve palsies are potential but rare complications of spinal anaesthesia. Most of the literatures support upper cranial nerve palsies like VI, IV and III cranial nerve palsies. Intrathecal hypotension resulting in tractional injury of the cranial nerves is the likely mechanism of injury. As on date, some cases of unilateral vocal fold paralysis and very little bilateral vocal fold paralysis have been described in case reports. We have described a patient who developed hoarseness and dysphagia 7 days after receiving spinal anaesthesia for fixation of inter-trochanteric fracture femur. The patient was diagnosed with bilateral vocal fold paralysis. He was managed conservatively and exhibited complete spontaneous recovery as has been described in the previously reported cases. Any patient presenting with idiopathic vocal fold paralysis should be enquired about the history of spinal or epidural anaesthesia. If the history is affirmative, then it points towards transient intrathecal hypotension as a potential etiology of the cranial nerve palsy.
\end{abstract}

Keywords: bilateral vocal fold palsy, cranial nerves, spinal anaesthesia, intrathecal hypotension.

DOI: $10.21303 / 2504-5679.2020 .001315$

\section{Introduction}

Spinal anaesthesia is a commonly used method of anaesthesia in lower limb and lower abdominal surgeries. Cranial nerve palsy is a recognized complication following spinal anaesthesia. The incidence of cranial nerve palsy following spinal anaesthesia described in older literature is 1 in 200 to 1 in 1,200 cases [1]. With the improvement of the standard of regional anaesthesia, the incidence decreased in recent decades. III, IV, V, VI, VII, VIII and X cranial nerve palsy have been described with VI cranial nerve palsy being the commonest [2-7]. Lower cranial nerve palsies are extremely rare and case reports are also minimal $[8,9]$.

\section{Case Summary}

Our patient was an 84 year of gentleman with long standing type 2 diabetes mellitus, ischemic heart disease with moderate LV systolic dysfunction (EF 48 \%) having a right sided inter-trochanteric fracture femur. He was posted for dynamic hip screw (DHS) fixation. He had an extremely kyphotic and scoliotic spine. Spinal anaesthesia was administered in the third attempt with 23 Gauge Quincke needle (after 2 attempts of failure with 25 Gauge Quincke needle).

Patient had developed hoarseness of voice and inability to cough, mild dysphagia and a headache that had lasted for 21 days. The symptoms started 7 days post-surgery. An initial ENT 
evaluation shortly after the start of the symptoms revealed bilateral vocal fold paralysis in flexible endoscopy. It was followed by CT Scan of Brain and spinal cord which were inconclusive. The patient had developed chest infection and was given a course of oral antibiotics and steroids. Though the chest infection improved, there was no improvement in speech and dysphagia. The patient was referred to a senior laryngologist. After 2 months, flexible endoscopy was done which revealed bilateral vocal fold paralysis with vocal folds fixed in intermediate position as well as a mild palatal weakness, indicative of high vagal injury. Conservative management continued and speech therapy was started. Videostroboscopy was done 4 months after the event, which revealed persistent bilateral vocal fold fixation in the intermediate position but slight movement of arytenoids towards the midline with maximal effort. Repeat flexible endoscopy, 6 months after the onset of symptoms, showed an almost normal fixation of the left vocal fold but only $50 \%$ function of the right vocal fold. Speech therapy continued along with supportive care including Ryles tube feeding. After 9 months of the first onset of symptoms, left vocal cord was normal and the right vocal cord was almost normal $(90 \%)$. The voice returned to baseline and dysphagia improved completely.

\section{Discussion}

Cranial nerve palsies, following spinal anaesthesia are a documented though rare complication. Most of the previously reported cases describe upper cranial nerve palsies like the sixth cranial nerve (commonest), oculomotor and trochlear neuropathies [2-6]. Facial and trigeminal neuropathies are also known to occur [7]. Recently, a few cases of lower cranial neuropathies have also been reported. A 2014 case series described 3 patients in whom unilateral vocal fold paralysis was noted up to 7 days after childbirth with spinal anaesthesia with a proposed mechanism of vagal neuropathy [9]. Reporting of bilateral vocal fold paralysis is however, extremely rare making this report unique.

A proposed pathophysiology of cranial neuropathies as a result of dural puncture has been described in the available literature. It is postulated that spinal-dural puncture leads to seepage of CSF which can last for many days. As a result, there is transient intrathecal hypotension leading to downward tractional injury of the cranial nerves at the level of the brainstem [2-8].

It is important for providers to be aware that the potential for permanent injury of cranial nerves exists in such cases. So, extreme safety measures should be undertaken in the course of administering spinal or epidural anaesthesia accordingly.

The patient in the presented case underwent spinal anaesthesia for a fixation of inter-trochanteric fracture femur with dynamic hip screw. Spinal anaesthesia was administered with 23 gauge Quincke needle as the patient's spine was kyphotic and scoliotic.

In these cases, headache is the commonest anaesthetic complication (80\%). In this case symptoms as described above started, 7 days after spinal anaesthesia, which was associated with mild headache. In our case, the patient started improving after six months and almost recovered $(90 \%)$ after 9 months. Symptoms improved after speech therapy, supportive care and improved nursing.

While bilateral vocal fold paralysis has the potential to cause critical airway compromise, the discussed patient was found to have focal fold fixation in the intermediate position [10]. This position allowed him to have an adequate airway and to be managed without the need for tracheostomy. Due to the transient nature of these injuries, it is important to counsel patients on the natural history and anticipated natural recovery as a part of their management. The counselling also needs to be done about the need for possible surgical intervention including tracheostomy and phonosurgery if spontaneous recovery does not happen. In case of bilateral vocal fold paralysis, injury to dura with resultant intrathecal hypotension should be considered where spinal anaesthesia was administered few days back.

\section{Conclusion}

Diagnostic workup of bilateral vocal fold paralysis following spinal anaesthesia should include proper history related to anaesthesia, flexible endoscopy, videostroboscopy and laryngeal EMG (if available) and CT or MRI of brain and spinal cord (if required). 


\section{Conflict of interest}

The authors declare there is no conflict of interest.

\section{Human ethics}

Every effort was made to preserve the privacy and confidentiality of the patient under discussion. The authors have ensured that consent is obtained from the patient for the said purpose.

\section{Reference}

[1] Robles, R. (1968). Cranial nerve paralysis after spinal anesthesia. Northwest Med, 67 (9), 845-847.

[2] Vandam, L. D. (1956). Long-term follow-up of patients who received 10,098 spinal anesthetics. Journal of the American Medical Association, 161 (7), 586-591. doi: http://doi.org/10.1001/jama.1956.02970070018005

[3] Whiting, A. S., Johnson, L. N., Martin, D. E. (1990). Cranial nerve paresis following epidural and spinal anesthesia. Trans Pa Acad Opthalmol Otolaryngol, 42, 972-973.

[4] Hofer, J. E., Scavone, B. M. (2015). Cranial Nerve VI Palsy After Dural-Arachnoid Puncture. Anesthesia \& Analgesia, 120 (3), 644-646. doi: http://doi.org/10.1213/ane.0000000000000587

[5] Basaranoglu, G., Saidoglu, L. (2013). Isolated transient diplopia and nystagmus after spinal anesthesia. Journal of Anesthesia, 27 (4), 643-644. doi: http://doi.org/10.1007/s00540-013-1575-y

[6] King, R. A., Calhoun, J. H. (1987). Fourth cranial nerve palsy following spinal anesthesia. Journal of clinical neuro-ophthalmology, 7, 20-22.

[7] Fang, J.-Y., Lin, J.-W., Li, Q., Jiang, N., Gao, Y. (2010). Trigeminal nerve and facial nerve palsy after combined spinal-epidural anesthesia for cesarean section. Journal of Clinical Anesthesia, 22 (1), 56-58. doi: http://doi.org/10.1016/j.jclinane.2009.01.016

[8] Castillo, M. (2002). Imaging of the upper cranial nerves I, III-VIII, and the cavernous sinuses. Magnetic Resonance Imaging Clinics of North America, 10 (3), 415-431. doi: http://doi.org/10.1016/s1064-9689(02)00009-0

[9] Guardiani, E., Sulica, L. (2014). Vocal Fold Paralysis Following Spinal Anesthesia. JAMA Otolaryngology-Head \& Neck Surgery, 140 (7), 662-663. doi: http://doi.org/10.1001/jamaoto.2014.721

[10] Dray, T. G., Robinson, L. R., Hillel, A. D. (1999). Idiopathic bilateral vocal fold weakness. The Laryngoscope, 109 (6), $995-$ 1002. doi: http://doi.org/10.1097/00005537-199906000-00028 